Editorial

\title{
Child mortality in the Eastern Mediterranean Region: challenges and opportunities
}

Zulfiqar A. Bhutta, MBBS, PhD, FRCP, FRCPCH, FCPS, FAAP'

In the year 2000, 189 heads of state signed the United Nations (UN) Millennium Declaration consisting of eight Millennium Development Goals (MDGs) with a focus on an integrated global agenda for poverty reduction, health, education and human development [1]. Although many countries have made progress, corresponding gains in targets for child survival (MDG4) and maternal survival (MDG5) are slow. It is estimated that to achieve MDG4 targets, the annual rate of decline in child mortality should be around $4.4 \%$, but the corresponding rate of decline since 1990 has only averaged $1.5 \%$ with wide variations between regions (Figure 1). Similarly, to achieve MDG5, the annual rate of maternal mortality decline needed to be $5.5 \%$ but global figures since 1990 indicate a decline of only $1.9 \%[2,3]$.

Around 39000 women of childbearing age still die every year in the World Health Organization Eastern Mediterranean Region (EMR) as a result of pregnancy-related complications (sepsis, haemorrhage, eclampsia, obstructed labour and unsafe abortions) [4]. Mortality rates are particularly high among pregnant adolescents who account for a significant proportion of first births. Overall the maternal mortality ratio (MMR) in EMR fell by 53\% between 1990 and 2010, and six countries have already achieved MMR beyond MDG5 targets and seven more are on track. Over $90 \%$ of the burden of maternal deaths in the Region is shared by seven countries: Afghanistan, Iraq, Morocco, Pakistan, Somalia, Sudan and Yemen. In terms of MDG5, five countries are not on track to achieve its targets: Djibouti, Iraq, Pakistan, Somalia and Tunisia [5].

In EMR $12.2 \%$ of the population comprises children under 5 years of age, while $20 \%$ are women of childbearing age. An estimated 923000 children under 5 years die every year in the Region and under-five mortality has decreased by only $41 \%$ since 1990 , from 99 deaths per 1000 live births to 58 per 1000 live births in 2011. EMR ranks fifth among the six WHO regions in terms of progress in reducing the under-five mortality, ahead only of the African Region [5].

Progress across the Region for child survival remains uneven. Six countries (Oman, United Arab Emirates, Bahrain, Lebanon, Qatar and Saudi Arabia) in EMR have already achieved reductions in the under-five mortality rate well beyond the targets of MDG4. The highest decrease in under-five mortality in the world (72\%) between 1990 and 2010 did occur in the Northern African countries of the Region. Of the total under-five deaths in EMR, 82\% occur in six countries (Afghanistan, Pakistan, Somalia, South Sudan, Sudan and Yemen). Of these, three countries, Sudan, Afghanistan and Pakistan, are among the 10 countries with highest child mortality in the world, a fact that has been recognized for almost a decade [6]. It is largely because of the high under-five population and mortality rates in these countries, which represent over half $(54 \%)$ of the total population in the Region, that EMR is unlikely to achieve MDG4.

We also know a fair bit about the major causes of child mortality in the Region and its determinants. Neonatal deaths represent a substantial proportion $(43 \%)$ of under-five mortality in the Region and are clearly linked to the poor status of maternal health among the poorest sections of the population [7]. In four countries, namely Afghanistan, Pakistan, Somalia and Yemen, less than $50 \%$ of deliveries were attended by skilled health personnel in 2010 [8]. Across the Region, only $31 \%$ of married women use modern contraceptives and $35 \%$ of women and newborns are delivered without a skilled attendant at childbirth. Beyond the neonatal period, four disorders - diarrhoea, pneumonia, malaria and measles - are the major causes of post-neonatal death [9].

These findings reinforce the imperative for action. Without intensive and accelerated action, especially in those countries contributing to the bulk of under-five deaths, the Region will not join the rank of developed countries and those that have climbed the development ladder. In recent months, commendable progress has been made in raising awareness in the Region and building the case for relevant evidence-based actions for change. A high-level meeting of health ministers, 


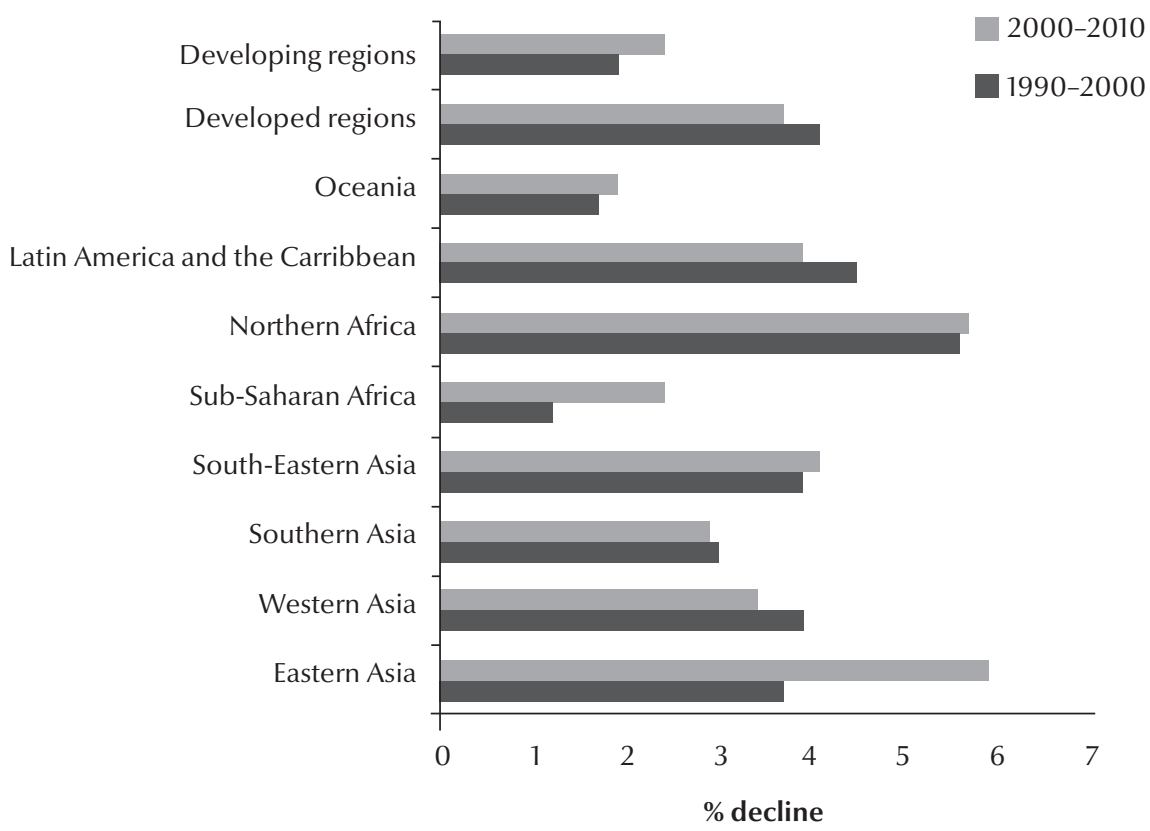

Figure 1 Trends in decline in under-5 mortality: Source: [1]

technical experts, academia and leading UN agencies was held in Dubai in January, 2013 and culminated in a consensus "Dubai Declaration" and a renewal of pledges for accelerated efforts. Four pillars were identified as critical to progress.

First, countries in the Region need to have reliable and robust information for action [10]. Not only does this relate to mortality trends and overall status, but disaggregated data highlighting differentials and vulnerable populations within countries is needed. Second, it was noted that evidence-based interventions existed but were failing to reach many who needed them most. These include not only the poorest sections of the population resident in difficult to access rural outposts but also those living in urban slums and marginalized on the basis of race or ethnicity. The monitoring process for coverage of essential interventions across the continuum of care spearheaded by Countdown for 2015 is one such initiative that could be used at the country level to assess progress and coverage [8]. Third, while interventions exist, there is a need to focus on delivery strategies and mechanisms for scaling up coverage in the short term. These include innovations for demand creation, removal of financial barriers as well as service delivery through community platforms and health workers who can reach the marginalized and poorest sectors of the population [11]. Finally, none of the above can be achieved without robust measures for monitoring and accountability. This must be done transparently and with the clear recognition that regular monitoring of gaps and remedial measures are critical to achieving equitable coverage. There is clear evidence that greatest gains in equity for coverage are in countries that have targeted scaling up universal coverage and deployed measures to reach the poor [12]. This cannot be done without information and targeted measures to address services in conflict-affected populations.

The challenge of wide differentials in maternal and child survival and life expectancy in EMR is real and a moral imperative to act for a Region rich in history, resources and a unifying faith that places social justice and rights of women and children at its core. We need to rise to the challenge and achieve such gains in equity for future generations, within a generation.

\section{References}

1. The Millennium Development Goals Report 2012. New York, United Nations, 2012.

2. Lozano R et al. Progress towards Millennium Development Goals 4 and 5 on maternal and child mortality: an updated systematic analysis. Lancet, 2011, 378:1139-1165.

3. United Nations Inter-agency Group for Child Mortality Estimation. Levels and trends in child mortality: Report 2012. New York,
United Nations Children's Fund, 2012 (http://www.childinfo. org/files/Child_Mortality_Report_2012.pdf, accessed 3 February 2013).

4. Lozano R et al. Global and regional mortality from 235 causes of death for 20 age groups in 1990 and 2010: a systematic analysis for the Global Burden of Disease Study 2010. Lancet, 2012, 380:2095-2128. 
5. Saving the lives of mothers and children: rising to the challenge Background document for the High Level Meeting on Saving the Lives of Mothers and Children: Accelerating Progress Towards Achieving MDGs 4 and 5 in the Region, Dubai, United Arab Emirates, 29-30 January 2013 (http://applications.emro. who.int/docs/High_Level_Exp_Reg_doc_2013_EN_14811.pdf, accessed 3 February 2013).

6. Bhutta ZA et al. Child health and survival in the Eastern Mediterranean region. British Medical Journal, 2006, 333:839-842.

7. Liu L et al. Global, regional, and national causes of child mortality: an updated systematic analysis for 2010 with time trends since 2000. Lancet, 2012, 379:2151-2161.

8. Countdown to 2012 Maternal, Newborn and Child Survival. Building a future for women and children. The 2012 Report. Geneva, World Health Organization and UNICEF, 2010.
9. Fischer-Walker $\mathrm{C}$ et al. Global burden of childhood diarrhoea and pneumonia: leading causes of child mortality. Lancet, 2013 (in press).

10. Every woman, every child: from commitments to action: the first report of the independent Expert Review Group (iERG) on Information and Accountability for Women's and Children's Health. Geneva, World Health Organization, 2012.

11. Lassi ZS, Haider BA, Bhutta ZA. Community-based intervention packages for reducing maternal and neonatal morbidity and mortality and improving neonatal outcomes. Cochrane Database of Systematic Reviews, 2010, (11):CD007754.

12. Victora CG et al. How changes in coverage affect equity in maternal and child health interventions in 35 Countdown to 2015 countries: an analysis of national surveys. Lancet, 2012, 380:1149-1156. 